\title{
HIF-1a Was a Key Regulator to Improve hBMSCs to Secrete Vascular Endothelial Cytokine with Astragaloside in Hypoxia
}

\author{
Ji Hong Hu ${ }^{1, ~ *}$, Jin Hua ${ }^{2}$, Jia Jia ${ }^{2}$, Lu Juan ${ }^{1}$, Jing Miao Zhao ${ }^{1}$, Shu Xia Wang ${ }^{1}$, Yun Wang ${ }^{1}$, \\ Li Mei Jin ${ }^{1}$, Jin Juan Li ${ }^{1}$ \\ ${ }^{1}$ Center of Research and Experiment, Public Health School, Gansu University of Chinese Medicine, Lanzhou, China \\ ${ }^{2}$ School of Chinese Medicine, Gansu University of Chinese Medicine, Lanzhou, China

\section{Email address:} \\ hujihonghappy@163.com (Ji Hong Hu), 505536382@qq.com (Jin Hua), 864241742@qq.com (Jia Jia), lujuan525@163.com (Lu Juan), \\ tcmzjm@163.com (Jing Miao Zhao),2649918377@qq.com (Shu Xia Wang),2429211711@qq.com (Yun Wang), \\ 15009426513@126.com (Li Mei Jin), 781281623@qq.com (Jin Juan Li) \\ *Corresponding author
}

\section{To cite this article:}

Ji Hong Hu, Jin Hua, Jia Jia, Lu Juan, Jing Miao Zhao, Shu Xia Wang, Yun Wang, Li Mei Jin, Jin Juan Li. HIF-1 $\alpha$ Was a Key Regulator to Improve hBMSCs to Secrete Vascular Endothelial Cytokine with Astragaloside in Hypoxia. American Journal of Clinical and Experimental Medicine. Vol. 7, No. 4, 2019, pp. 75-82. doi: 10.11648/j.ajcem.20190704.11

Received: August 1, 2019; Accepted: September 6, 2019; Published: September 23, 2019

\begin{abstract}
This study aimed to assess the combined therapeutic efficacies with gene therapy and stem cell treatment and herb on the differentiation of hBMSCs into vascular endothelial cells under hypoxia condition. The third passage of hBMSCs were randomly divided into four groups, including control group (hBMSCs transfected with empty vectors), VEGF group (VEGF gene transfected to hBMSCs), AST group (cultured with AST), and VEGF + AST group (the intervention of VEGF group plus AST group). Each group was cultured at $37^{\circ} \mathrm{C}$ and $5 \% \mathrm{O}_{2}$ for 2 weeks. Cell morphology was observed by inverted phase and were crowded and arranged irregularly in the control group and AST group, showing a fiber-like growth, while those in the VEGF group and VEGF plus AST group were mostly triangular or polygonal, exhibiting a colony-like growth, contrast microscope. CD31 was negative in the control group and AST group, while CD105 was positive in both groups, tested by flow cytometry assay. The positive rate of CD31 was significantly higher in the VEGF group than it in the VEGP + AST, and the positive rate of CD105 was lower in the VEGF group than it in the VEGF + AST group. The levels of VEGF and endothelial nitric oxide synthase (eNOS) by ELISA and the expression of endothelin and prostacyclin by west blot (WB) and RT-PCR were significantly higher in VEGF group and AST group and VEGF plus AST group, compared with control group. Further, the expression of endothelin and VWF and VEGFR-2 was highest in the VEGP + AST group and the expression of prostacyclin was lowest in VEGF group. AST can promote the secretion of VEGF from the differentiation of hBMSCs induced by VEGF gene transfected to hBMSCs by HIF-1 $\alpha$ under hypoxia.
\end{abstract}

Keywords: HIF-1 $\alpha$, Astragaloside, Hypoxia, Bone Mesenchymal Stem Cells, VEGF Gene Transfection, Vascular Endothelial Cytokine

\section{Introduction}

Cardiovascular disease (CVD) is the leading cause of death both in the developing and developed countries and constitutes a major public health problem. The rising trend of cardiovascular mortality mainly results from the rising death rate of ischemic heart disease [1]. Therapeutic angiogenesis refers to actions performed to facilitate revascularization of ischemic tissues. Bone marrow stem cells (BMSCs) have been shown to become ideal stem cells for therapeutic angiogenesis due to self-renewal, multi-trans differentiation and paracrine effects, especially secreting a variety of vascular growth factors, compared with other stem cells [2-4]. However, BMSCs are faced with a hypoxic-ischemic microenvironment after implantation into a myocardial ischemic area. A critical 
question that emerges is how to utilizeparacrine cytokines to reconstruct a collateral microcirculation as part of the process to repair the myocardium.

At present, there is no clear induction agent for directional differentiation of BMSCs to VECs [30]. Members of the vascular endothelial growth factor (VEGF) family are the strongest known modulators of vascular biology. They regulate vasculogenesis, angiogenesis, and vascular maintenance during embryogenesis and in adults. Co-culture of VEGF and BMSCs can induce BMSCs to differentiate into vascular endothelial cells [5]. Our previous study has found that BMSCs transfected by a VEGF gene can differentiate into vascular endothelial cells in a hypoxic environment [6]. However, completed randomized controlled VEGF trials using VEGF delivered as are combinant protein or via gene transfer for the treatment of myocardial or peripheral ischemia have not provided convincing evidence of clinical efficacy, primarily because of their rapid elimination in vivo [7].

A large number of animal and clinical studies have found that Astragalusmembranaceus can significantly improve cardiac function, reduce myocardial infarction area, reduce tissue impairment and inhibit cognitive impairment [8-11].

Astragaloside IV (AST) is the major active saponin abundant in Astragalusmembranaceus and, reportedly, has a variety of pharmacological activities. AST had a significant effect on the treatment of ischemic heart disease [12-16], protected BMSCs from apoptosis and promoted proliferation in vitro [11]. Consequently, it is hypothesized that the combination treatment of BMSCs with VEGF gene transfection and AST will improve the differentiation from BMSCs into vascular endothelial cells and their subsequent proliferation.

\section{Materials and Experiments}

\subsection{Main Instruments and Reagents}

The main instruments and reagents listed in Table 1. Primers were synthesized by the Invitrogen Corporation. AST (purity quotient of 99.8\%) were purchased from National Institutes for Food and Drug Control (batch No. 100781200613). 20mg AST was added into $200 \mu \mathrm{L}$ dimethyl sulfoxide (DMSO), then gently mixed, then added to DEM to $50 \mathrm{~mL}$ and completely dissolved, filtered by filter membrane with a pore size of $0.22 \mu \mathrm{m}$, resulting in a mother liquor $(0.4 \mathrm{mg} / \mathrm{mL})$ which was saved at $20^{\circ} \mathrm{C}$.

Table 1. Cells, reagents and instruments.

\begin{tabular}{ll}
\hline Cells, reagents and instruments & corporation \\
\hline $\begin{array}{l}\text { Human bone marrow mesenchymal stem } \\
\text { cells (1st generation) }\end{array}$ & $\begin{array}{l}\text { Scien Cell corporation (Cargo } \\
\text { No. 7500) }\end{array}$ \\
$\begin{array}{l}\text { MSCM culture medium and fetal bovine } \\
\text { serum }\end{array}$ & $\begin{array}{l}500 \mathrm{~mL} \text {, Scien Cell } \\
\text { corporation }\end{array}$ \\
$\begin{array}{l}\text { Adenovirus vector pAd- vegf-ires2-egfp } \\
\text { (293A cells) }\end{array}$ & Life Technologies Corporation \\
The viral titer is 2 x 1010 ifu/ml & \\
TakaRa Prime ScriptTM reagent Kit & TaKaRa corporation \\
SDS-PAGE gel preparation kit & Solarbio Corporation \\
CD31 FITC、CD105 APC & BD Corporation \\
\hline
\end{tabular}

\begin{tabular}{ll}
\hline Cells, reagents and instruments & corporation \\
\hline \multirow{2}{*}{ Human VEGF ELISA kit } & Consortium creatures, batch \\
& no:218360343 \\
Human eNOS ELISA kit & Shanghai elisa, batch \\
& no:201604 \\
BCA protein concentration assay kit & Solarbio Corporation \\
inverted phase contrast microscope & OLYMPUS Corporation \\
flow cytometry & BD FAC Scan Corporation \\
\hline
\end{tabular}

\subsection{Human BMSCs Culture}

The human BMSCs were propagated from generation 1 to 3 in a $37^{\circ} \mathrm{C}, 5 \% \mathrm{CO}_{2}$ incubator. When the cell confluence reached $80 \%$ to $90 \%$, they were transferred to a $37^{\circ} \mathrm{C}, 5 \% \mathrm{O}_{2}$ incubator for differentiation. All experimental tests were performed after 2 weeks incubation.

The third-generation BMSCs were randomly divided into 4 groups as follows: Normal control group (control group): DMEDM cultured for 2 weeks; VEGF induction group: DMEDM culture containing Adenovirus vector pAd-VEGF-IRES2-EGFP [5]; AST group: DMEDM culture containing AST [7]; VEGF + AST group: combination of interventions in the VEGF group and in the AST group. After 3 days induction, the VEGF group, AST group and VEGF + AST group were changed into a common DMEDM culture condition. All groups were renewed with DMEDM culture medium every 3 days and saved the culture supernatant was saved and stored at $-20^{\circ} \mathrm{C}$, for a period of two weeks of culture.

\subsection{Characterization of Vascular Endothelial Cells (VECs) Phenotype by Flow Cytometry}

Cells were collected at differentiation day 0 and at 2 weeks. After trypsin-digestion and centrifugation, the cells were incubated with anti-human CD31 (PECAM-1), a marker of white cells and endothelial cells and anti-human CD105 (endoglin), a marker of actively proliferating endothelial cells and angiogenesis (BD Inc, USA). Subsequently, the expression of CD31 and CD105 on the cell surface was analyzed by flow cytometry.

\subsection{Detection of VEGF and Endothelial Nitric Oxide Synthetase (eNOS) in Supernatants}

At the end of 2 weeks, the expression levels of VEGF and eNOS in supernatants were detected using VEGF and eNOS kits with ELISA according to manufacturer recommendations.

\subsection{Detection of Endothelin (ET) and Prostacyclin (PGI) by Real-time qPCR}

After 2 weeks of induction and culturing, the cells were collected and the total RNA was extracted and reverse transcribed to cDNA according to the instructions of the TakaRa Prime ScriptTM reagent Kit. Primers are shown in Table 2. Real-time PCR was undertaken as follows: $1 \mu \mathrm{g}$ RNA was added to $4 \mu \mathrm{L} 5 \times$ PrimeScript Buffer and $1 \mu \mathrm{L}$ PrimeScript RT Enzyme Mix I and $1 \mu \mathrm{L}$ Oligo dT Random Primer and $1 \mu \mathrm{L}$ 6 mers. Then, RNase Free $\mathrm{dH}_{2} \mathrm{O}$ was added to $20 \mu \mathrm{L}$. Lastly, the samples were amplified at $37^{\circ} \mathrm{C}$ for $15 \mathrm{~min}, 85^{\circ} \mathrm{C}$ for $5 \mathrm{~s}$, 
the $4^{\circ} \mathrm{C}$ for $10 \mathrm{~min}$. Real-time PCR was used to test the cDNA obtained from reverse transcription and make the dissolution curve.

The $\mathrm{Ct}(\mathrm{Ct})$ was used to calculate the relative gene expression data using Real-Time Quantitative PCR.

Table 2. Sequence of primers detected by $q P C R$.

\begin{tabular}{ll}
\hline primer sequence \\
\hline \multirow{2}{*}{ ET } & $\begin{array}{l}\text { forward primer:5' -TGC TCC TGC TCG TCC CTG ATG GAT } \\
\text { AAA GAG-3' } \\
\text { downstream primers: 5' -GGT CAC ATA ACG CTC TCT GGA } \\
\text { PGI }\end{array}$ \\
$\begin{array}{l}\text { GGG CTT-3' } \\
\text { orwardprimer:5' -ACC CCC CAC TGA AAA AGA TGA-3' } \\
\text { downstream primers:5'-CCT TCT AAG TGG TTG GAACA-3' } \\
\text { GADPH }\end{array}$ & $\begin{array}{l}\text { orward primer:5'-TGA AGG TCG CTG TCA ACG GA-3' } \\
\text { downstream primers:5'-GAT GGC ATG GAC TGT GGT } \\
\text { CAT-3 }\end{array}$ \\
\hline
\end{tabular}

\subsection{Detection of ET and PGI2 Proteins in Cells by Western Blotting}

After 2 weeks of induction and culturing, the cells were collected, pyrolysis solution was added and placed on ice for $10 \mathrm{~min}$. After $12000 \mathrm{rpm}$ centrifugation for $30 \mathrm{~min}$ at $4^{\circ} \mathrm{C}$, the solution was extracted and the protein concentration was measuredusing the BCA method. The protein was added to $7.5 \%, 9 \%$ gel for SDS-PAGE, and transferred using $200 \mathrm{~mA}$ constant flow under wet conditions to PVDF membrane. Antibody I was incubated at $4{ }^{\circ} \mathrm{C}$ overnight, and then incubated with the correspondingantibody IIat room temperature. Thereafter, exposure was performed using an electrochemiluminescence solution (ECL). ImageJ software analyzes were undertaken using the grayscale values of the strips.

\subsection{Immunocytochemistry (ICC) for vWF, HIF-1 $\alpha$ and VEGFR-2 After 7 Days}

The expression of vWF, HIF-1 $\alpha$ and VEGFR-2 was determined after 7 days. Cells were cultured on glass coverslips, washed with PBS 3 times. then naturally dried, cells were fixed in $4 \%$ poly formaldehyde for $30 \mathrm{~min}$ and then immersed in PBS and washed by PBS $5 \mathrm{~min} \times 3$ times. Then, cells were infiltrated with $0.1 \%$ Triton $\mathrm{X}-100$ for $15 \mathrm{~min}$, and then washed by PBS $5 \mathrm{~min} \times 2$ times. Furthermore, cells were blocked for endogenous peroxase-activity for $10 \mathrm{~min}$, and then washed by PBS $5 \mathrm{~min} \times 3$ times again, and were blocked for $10 \mathrm{~min}$ and flushed by PBS $5 \mathrm{~min} \times 3$ times. Cells were incubated with the mouse monoclonal anti-VWF (1:100), anti-VEGFR-2 (1:100), and anti HIF-1 $\alpha$ (1:100) for $60 \mathrm{~min}$. After thorough washing with PBS, secondary antibodies of goat anti-mouse IgG and/or goat anti-rabbit IgG were applied for $10 \mathrm{~min}$ incubation, and then flushing by PBS $5 \mathrm{~min} \times 3$ times. Next, cells were incubated with Streptavidin-HRP working liquid for $10 \mathrm{~min}$ and washed by PBS $5 \mathrm{~min} \times 3$ times. Finally, DAB' staining and Sumusu' re-dyeing and dehydration were performed. Fluorescent images were obtained using an Olympus DP70 microscope equipped with a digital camera (Olympus). Cells were examined randomly using 5 microscopes' FOV (field of vision) and imaged at 200x magnification. The positive rate was the ratio of positive cells to total cells.

\subsection{Statistical Methods}

SPSS V13.0 statistical software was used for analysis. The Kolmogorov-Smirnov test was used for tests of normality. The measurement data with normal distribution were described as mean \pm standard deviation (SD). The differences among groups were compared with one-way variance analysis, and LSD was further used for pair-wise comparison between any two groups. If the data were a non-normal distribution, the data were expressed as median and quartiles and the Kruskal-Wallis $\mathrm{H}$ test was used for comparison between groups. $\alpha=0.05$.

\section{Results}

\subsection{Morphology of BMSCs with Inverted Phase Contrast Microscope}

As shown in Figure 1, BMSCs presented spindle-shaped, oval nuclei, fibrous growth and vigorous growth in the normal control group (Figure 1A). After 2 weeks of differentiation, the number of cells in the AST group was increased, which were still spindle shaped and interwoven, without obvious morphological changes (Figure 1B). The cells in the VEGF group and VEGF + AST group were mostly triangular or polygonal, were growing in colonies and were confluent (Figure 1C, Figure 1D).

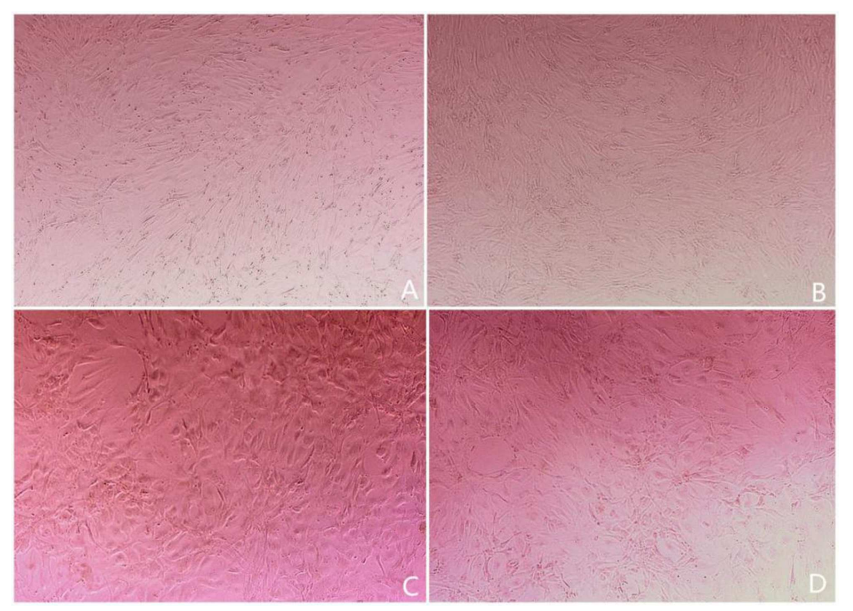

A: Control group, B: AST group, C: VEGF group, D: AST + VEGF group.

Figure 1. Morphological observation of each group at 14 days after induction under inverted microscope $(4 \times)$.

\subsection{Detection of Cell Surface Markers by Flow Cytometer}

After 2 weeks of differentiation, the cell surface marker CD31 was negative in the AST group and the control group, however, CD105 was positively expressed, with a respectively positive rate of $99.7 \%$ and $99.6 \%$, indicating that the BMSCs phenotype was still present in the cells (Figure 2A and Figure 2B). CD31 expression was significantly higher in both the VEGF and AST + VEGF 
group than it in both control group and AST group, furthermore, the positive rate in the VEGF group was higher than that in the AST + VEGF group (30.33\% vs. $10.22 \%$,

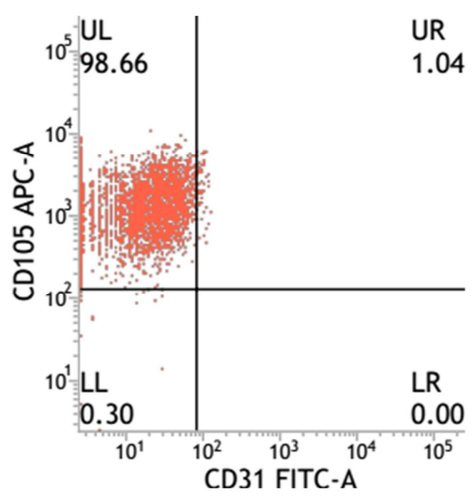

A

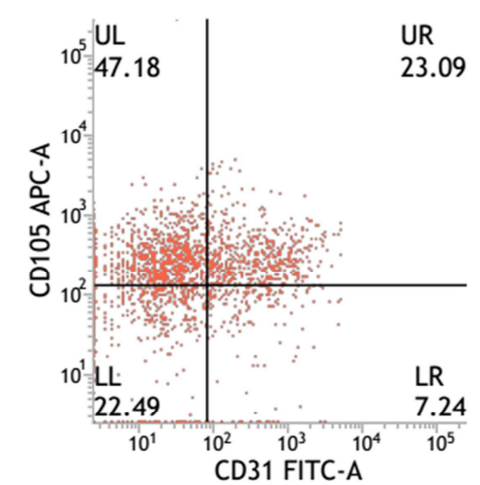

C

A: Control group; B: AST group; C: VEGF group; D: AST + VEGF group.
$P<0.05)$. CD105 expression in VEGF group was significantly lower than that in the AST + VEGF group (58.11\% vs. $63.47 \%, P<0.05$ ) (Figure $2 \mathrm{C}$ and Figure $2 \mathrm{D}$ ).

B
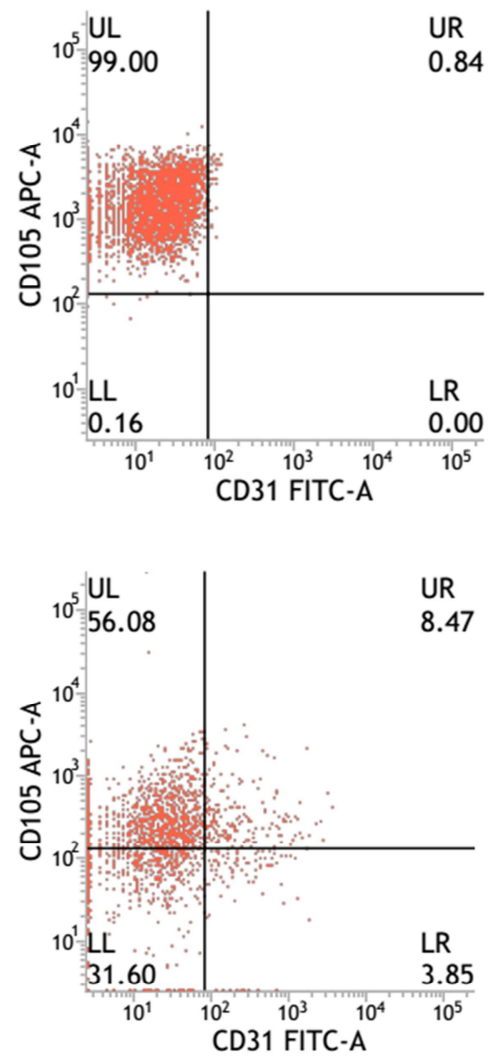

D

Figure 2. Expression of CD31 and CD105 in hBMSCs after 2 weeks induction.

\subsection{Expression of ET $m R N A$ and PGI $m R N A$ by RT-PCR}

Compared with the control group, ET mRNA in the three intervention groups were significantly higher (all $\mathrm{P}<0.05$ ), whereas PGI mRNA were lower (all $P<0.05$ ) (Table 3). Compared with the AST group, ET mRNA in VEGF group and VEGF + AST group both were significantly higher, however PGI mRNA were lower (all $P<0.05$ ). Compared with the VEGF group, ET mRNA was significantly lower in the AST + VEGF group $(P<0.05)$, while PGI mRNA was increased $(P<0.05)$, as shown in table 3 .

Table 3. Expression of ET and PGI $m R N A$ after 2 weeks induction (mean $\pm S D, n=3$ ).

\begin{tabular}{llll}
\hline & Control group & AST group & VEGF group \\
\hline GAPDH & $16.12 \pm 0.02$ & $15.66 \pm 0.02$ & $15.43 \pm 0.03$ \\
ET & $28.05 \pm 0.28$ & $26.45 \pm 0.03$ & $25.12 \pm 0.07$ \\
PGI & $26.84 \pm 0.21$ & $27.38 \pm 0.01$ & $27.93 \pm 0.01$ \\
ET 2- $\Delta \Delta$ Ct & $1.00 \pm 0.26$ & $2.21 \pm 0.06^{* \#}$ & $4.71 \pm 0.24^{* \mathcal{O}}$ \\
PGI 2- $\Delta \Delta$ Ct & $1.00 \pm 0.20$ & $0.39 \pm 0.00^{* \#}$ & $0.29 \pm 0.00^{* \mathcal{O}}$ \\
\hline
\end{tabular}

*Compared with the control group, $\mathrm{P}<0.05$; CCompared with the AST group, $\mathrm{P}<0.05$; \#compared with VEGF group, $\mathrm{P}<0.05$.

\subsection{Expression of ET and PGI Proteins by Western Blotting}

$\beta$-actin was expressed in each group. Compared with the control group, ET protein was expressed significantly higher in the VEGF group, and the AST group and the VEGF + AST group (all $P<0.05$ ), while PGI was lower (all $P<0.05$ ) in VEGF group and AST group and VEGF + AST group (Figure
3). Compared with the AST group, levels of ET protein were significantly higher in both VEGF group and VEGF + AST group (both $P<0.05$ ), while PGI was lower (both $P<0.05$ ). Compared with VEGF group, both levels of ET and PGI were significantly higher in the VEGF + AST group (both $P<0.05$ ) (Shown in Figure 3). 


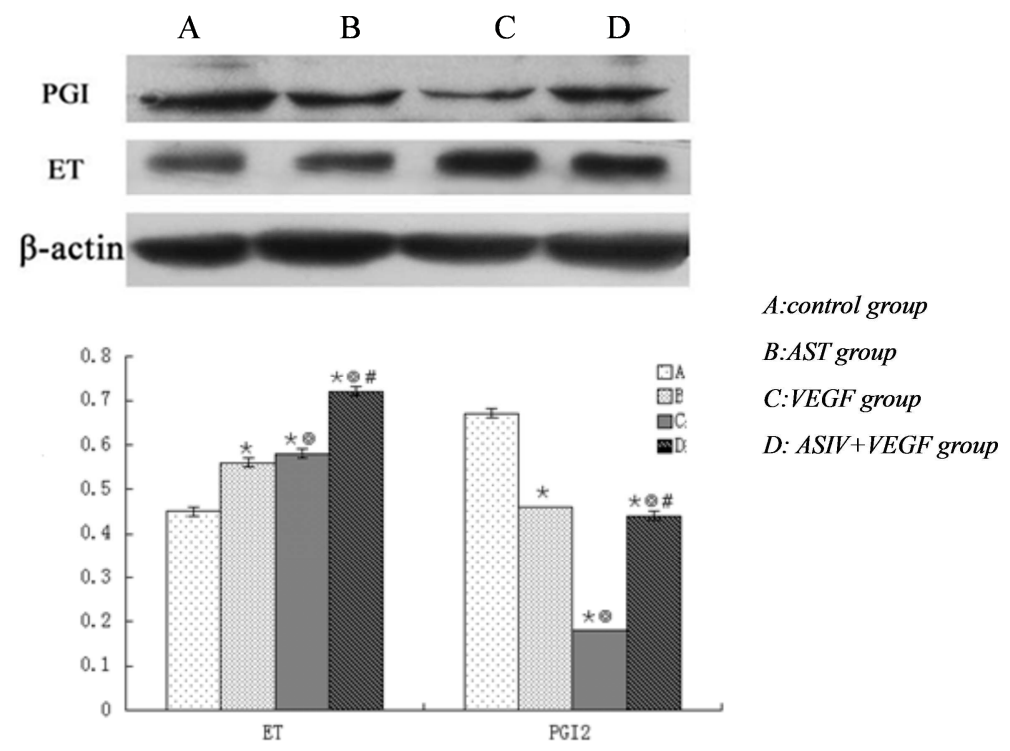

*Different to control group, $\mathrm{P}<0.05$; ○different to AST group, $\mathrm{P}<0.05$; \# different to VEGF group, $\mathrm{P}<0.05$.

Figure 3. Expression of ET and PGI proteins in each group after 2 weeks under hypoxia.

\subsection{Expression of VEGF and eNOS by ELISA}

Compared with the control group, the levels of VEGF and eNOS were significantly higher in the VEGF group and the AST group and VEGF + AST group (all $P<0.05$ ). Compared with the AST group, the expression levels of VEGF and eNOS were significantly higher in both the VEGF group and the VEGF + AST group (all $P<0.05$ ). Compared with the VEGF group, the expression levels of VEGF and eNOS were significantly higher in the VEGF + AST group (both $P<0.05$ ) (seen in Table 4.).

Table 4. Expressions of VEGF and eNOS in supernatants after 2 weeks induction (mean $\pm S D, n=4)$.

\begin{tabular}{llll}
\hline & Control group & AST group & VEGF group \\
\hline VEGF $(\mathrm{pg} / \mathrm{mL})$ & $450.99 \pm 18.07$ & $700.10 \pm 39.45^{* \#}$ & $976.50 \pm 171.95^{* \mathcal{C}}$ \\
eNOS $(\mathrm{U} / \mathrm{mL})$ & $21.13 \pm 0.94$ & $24.01 \pm 0.74^{* \#}$ & $27.40 \pm 1.14^{* \mathcal{C}}$ \\
\hline
\end{tabular}

*Compared with control group, $\mathrm{P}<0.05$; Ccompared with AST group, $\mathrm{P}<0.05$; \#compared with VEGF group, $\mathrm{P}<0.05$.

\subsection{The Positive Rate of Von Willebrand Factor (vWF), VEGFR-2 and HIF-1a by ICC}

Compared with the control group, the levels of vWF and VEGFR-2 were significantly higher in the VEGF group and the AST group and VEGF + AST group (all $P<0.05$ ); the levels of HIF-1 $\alpha$ were significantly higher in AST group and AST + VEGF group (both $P<0.05$ ). Compared with AST group, VEGFR-2 was significantly higher in AST + VEGF group $(P<0.05)$; the levels of vWF was significantly higher in both VEGF group and AST + VEGF group (both $P<0.05$ ). Compared with VEGF group, the expression levels of vWF and VEGFR-2 both were significantly higher in AST + VEGF group (both $P<0.05$ ) (Figure 4 ).

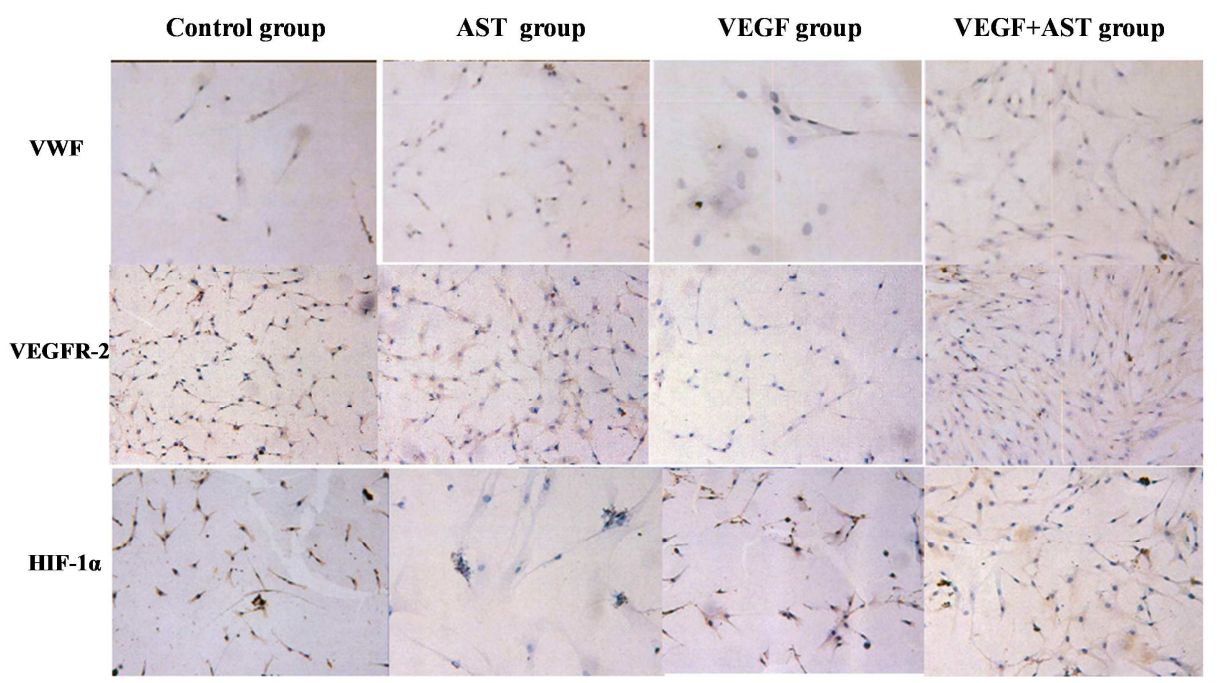




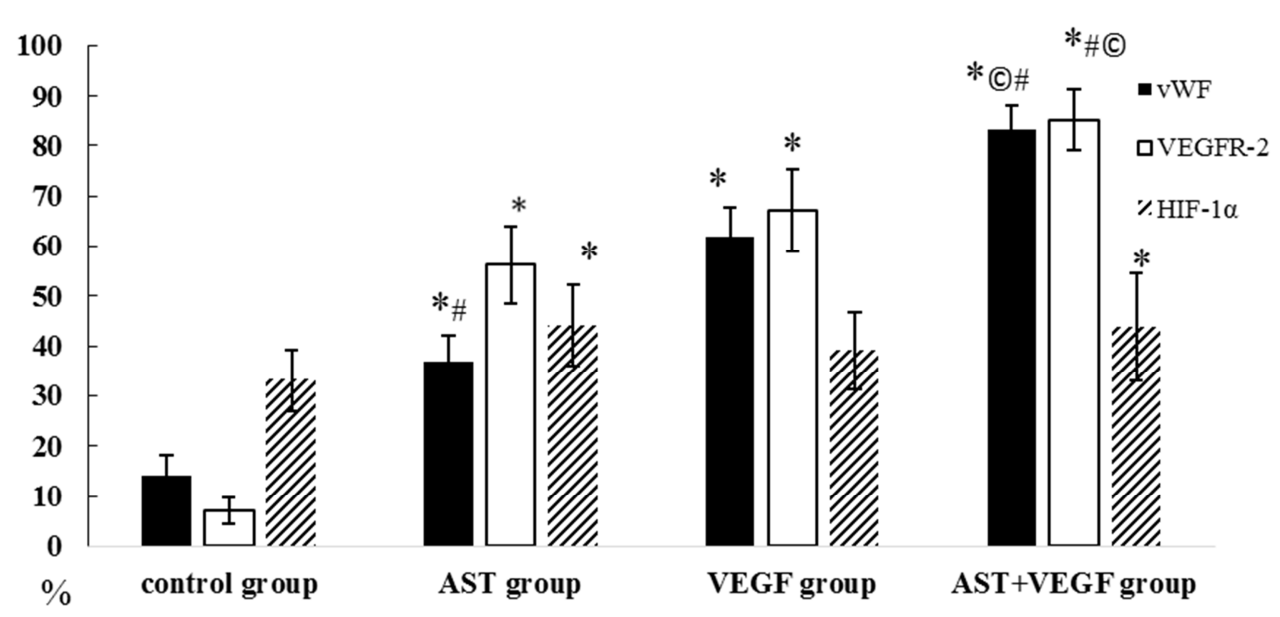

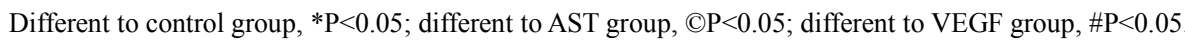

Figure 4. The positive rate of $v W F, V E G F R-2$ and HIF-1 $\alpha$ after 7 days induction.

\section{Discussion}

Ischemic diseases (such as cerebral embolism, myocardial infarction, osteonecrosis, etc.) result in local tissue ischemia and hypoxia. Therapeutic angiogenesis stimulates the formation and maturation of new blood vessels by introducing pro-angiogenic growth factors, thereby increasing blood perfusion, reducing tissue damage and promoting repair. BMSCs have the ability to undergo multidirectional differentiation. BMSCs can be differentiated into corresponding mature cells by specific inductions, and then are able to repair corresponding tissues at the injury sites which are mostly ischemic and hypoxic [12]. Thus, BMSCs become seed cells for cell therapy and gene therapy. After stem cell shave been transplanted, most stem cells grow within the microenvironment of tissue hypoxia. Thus, how to promote the survival and directional differentiation of BMSCs is the focus of many researchers. VEGF has been considered as the most effective vascular growth promotion factor until now [13]. Our previous study has found that VEGF gene transfection into human BMSCs induced BMSCs differentiation into VECs under hypoxic conditions [5]. AST is the main active component of Astragalus. It has been reported that AST not only increases the proliferation of human BMSCs in vitro, but also increases the expression of VEGF mRNA [14]. However, the role of a combination of VEGF gene transfection and AST treatment, to facilitate more effective differentiation into functional VECs and increased proliferation under hypoxic conditions, remains to be evaluated.

In this study, the cells in VEGF group and VEGF + AST group presented the phenotypic characteristics of endothelial cells, and VEGF transfection probably had a tendency to differentiate into VECs in hypoxia. This is consistent with the results of human BMSCs differentiation to endothelial cells induced by VEGF transfection under normal oxygen [15]. It also was found that the co-culture of human BMSCs [17-18], mouse BMSCs [19] or sheep BMSCs [20] with VEGF also promoted the differentiation of BMSCs into endothelial cells under normal oxygen conditions. However, AST did not promote the differentiation of human BMSCs into VECs. The result is different from that CD31 positively expressed after BMSCs induced by Astragalus injection [21]. The differences may be due to other active components in Astragalus promote the differentiation of BMSCs into VECs. Additionally, we found that the different test time probably resulted in the different ranks of CD31, and vWF and VEGFR-2 among 4 groups. It was reported that VEGFR-2 firstly expressed, subsequently CD31 and vWF expressed in BMSCs; VEGFR-2 regulated the budding of capillary growth and played a main role in angiogenesis [29].

Mature VECs eventually play their functions through the related cytokines which are classified into endothelium-derived contraction factors (eg, ET and thrombatin, etc.) and diastolic factors (eg, NO and PGI, etc.). If or not the differentiated human BMSCs have the functions of VECs, it is necessary to verify the related functional cytokines had positive or negative expression. At the present, the relevant study is rare. Our previous study found that VEGF gene transfection increased ET and reduced PGI under hypoxia [5]. In this study, ET, ET mRNA, eNOS and VEGF all upregulated, but PGI and PGI mRNA downregulated in VEGF group and AST group and VEGF + AST group, moreover, the promotion power is different. Normal VECs can maintain homeostasis and play function [24] and promote the normal growth of VECs [25] only when the secretion of ET increases and PGI reduced. VEGF is currently known as the strongest and most specific angiogenic factor, which plays an important role in maintaining the normal structure and physiological function of VECs [22]. eNOS produces nitric oxide (NO) in vascular vessels and helps to regulate vascular function. It was found that BMSCs expressed the cytokines related with VECs such as CD31 and VEGF after the endothelium-type eNOS vector is transfected to BMSCs [23]. In addition, AST improved the VEGF expression of BMSCs although AST not had the potential directional induction ability of human BMSCs differentiation into VECs in this study. The result is consistent with the previous studies $[14,21]$. We also found 
that ET was highest in AST + VEGF group. AST is often used as a complementary and alternative medicine which can provide $Q i$ in clinical practice. The results in this study conforms to the theory of "Qi produces blood" in Chinese medicine.

HIF-1 $\alpha$ is as a key regulator of the molecular hypoxic response and a master regulator of the cellular homeostatic response to hypoxia by activating many genes, including those involved in energy metabolism, angiogenesis, apoptosis and so on [31]. In this study, HIF-1 $\alpha$ was upregulated in AST group and AST + VEGF group. It is consistent with the results in ischemia and reperfusion injury [32]. Hypoxia increases VEGF-1 expression via up-regulation of HIF-1 $\alpha$ [33-34]. However, VEGF was highest in VEGF group, but not AST + VEGF. The reasons need further study to explore.

There are some limitations in this study. Firstly, we not designed the comparison between hypoxia and normal oxygen. Secondly, we mainly did test at the end of experiment. The continuous changes of all test variables can't be observed and analyzed. Thirdly, although AST not induced BMSCs to differentiate into VECs, it could promote BMSCs to secrete cytokines of VECs. The mechanism of these problems needs to explore.

\section{Conclusion}

In hypoxic, HIF-1 $\alpha$ probably plays a key positive regulatory role in which AST promotes the secretion of vascular endothelial cell-associated factors by hBMSCs, including the increase expression of ET, PGI, VEGF and eNOS. Additionally, via HIF-1 $\alpha$, AST combined with VEGF can promote the secretion of ET and $\mathrm{vWF}$ and VEGFR-R2 by VECs-like cells. It suggests that HIF-1 $\alpha$ master AST promoting the differentiation of BMSCs into vascular endothelial cells by angiogenesis regulation to hypoxia.

\section{Acknowledgements}

All authors of this research paper have no conflicts of interest to disclose. The corresponding authors of this manuscript are Jihong $\mathrm{Hu}$, and contribution of the authors as mentioned below with their responsibility in the research. All authors of this research paper have directly participated in the planning, execution, or analysis and interpretation of data and have read and approved the final version submitted. The contribution of each author is as follows: Jihong $\mathrm{Hu}$ (PhD.) were involved in conceptualization and drafted the manuscript. Jin Hua (PhD.), JiaJia (M. S), Lu Juan (M. M), Jing-Miao Zhao (M. M), Shu-Xia Wang (B. M), Yun Wang (B. M) conducted the experiment. Li-Mei Jin (MM) and Jin-Juan Li inputted and analyzed. This experiment was performed in Research Center of Gansu University of Chinese Medicine. This work was funded by the National Natural Science Foundation of China.

\section{References}

[1] Hui Sui, Weiwei Chen, Wen Wang. "China cardiovascular disease report 2014". Chinese journal of hypertension. 2015; 23 (7): 627-629.

[2] Chen X, Kata Kowskim, Li Y, et al. Human bone marrow stromal cell cultures conditioned by traumatic brain tissue extracts: growth factor production [J]. Neurosci Res. 2002; 69 (5): 687-691.

[3] Tomita S, Mickle DA, Weisd RD, et al. Improved heart function withmyogenesis and angiogenesis after autologous porcine bone marrow stromalcell transplantation [J]. J Thora Cardiovasc Surg. 2002; 123 (6): 1132-1134.

[4] Haiyan Feng, Ruifeng Liu, Kaiming Zhang, et al. In vitro isolation and culture of human bone marrow mesenchymal stem cells and differentiation into vascular endothelial cells [J]. Chinese journal of immunology. 2010; 26 (1): 70-74.

[5] Jihong $\mathrm{Hu}$, Jia Jia, Juan Lu, Qiuping Wang, Jingmiao Zhao. Differentiation of human bone marrow mesenchymal stem cells into vascular endothelioid cells transfected with vascular endothelial growth factor under hypoxia [J]. Tissue engineering research in China, 2017; 21 (9): 1352-1356.

[6] DongSei, Shuqing Li, Donghua Li, et al. Effects of astragalus on improving cardiovascular function [J]. Chinese clinical rehabilitation. 2006; 10 (11): 21-23.

[7] ZHANGJin-guo, GAO Dong-sheng, YANG Na, et al. Effect of astragalus injection on left ventricular remodeling and cardiac function in early morning patients with acute myocardial infarction [J]. Chinese Journal of Integrative Medicine. 2003; 9 : 104-107.

[8] LIU Yong-qi, LI Jing-ya, CAI Ling, et al. Differentiation of rat bone marrow mesenchymal stem cells induced by astragalus polysaccharide $[\mathrm{J}]$. Chinese journal of traditional Chinese medicine. 2014; 21 (6): 60-64.

[9] Shaoxian Xian, Zhongqi Yang, Zhaohui Wang, et al. In vitro induction of astragaloside iv into myocardial differentiation of bone marrow mesenchymal stem cells [J]. Journal of guangzhou university of traditional Chinese medicine. 2007; 24 (1): $37-40$

[10] Lin Liu, Hai Hai, Cun bang Wang, et al. Effect of astragalus polysaccharide on proliferation and cytokine expression of bone marrow mesenchymal stem cells in patients with multiple myeloma [J]. Advances in modern biomedicine. 2013; 13 (10): 1873-1877.

[11] Jihong Hu, Juan Lu, Yi Zhao, Limei Jin, Li Yan, Jia Jia, Qiuping Wang, Jingmiao Zhao. Effects of astragalus major active ingredients and extracts on proliferation of rat bone marrow mesenchymal stem cells [J]. Lishizhen Medicine and Materia Medica Research. 2016; 5: 1070-1072.

[12] Muschler GF, Nakamoto C, Griffith LG. Engineering principles of clinical cell-based tissue engineering [J]. J Bone Joint Surg AM. 2004; 86: 1541-1558.

[13] Junhua Song, Shuyun Wang. Effect of VEGF gene on ischemic heart disease and its influencing factors [J]. Journal of binzhou medical college. 2004; 27 (4): 281-284. 
[14] Xiaojuan Peng, Hai Bai, Cun-bang Wang, Rui Xi, et al. Effect of astragaloside iv on proliferation and cytokine expression of human bone marrow mesenchymal stem cells in vitro [J]. Advances in modern biology. 2014; 8: 1452-1455.

[15] Xuelan LUO, Wei CHEN, Guojun MO, et al. The difference of eNOS expression/ activity and its metabolites in human bone marrow-derived mesenchymal stem cell-oriented vascular endothelial cells [J]. Journal of Xi'an Jiaotong University. 2017; (3): 386-391.

[16] Irina Arutyunyan, TimurFatkhudinov, Evgeniya Kananykhina, et al. Role of VEGF-A in angiogenesis promoted by umbilical cord-derived mesenchymal stromal/stem cells: in vitro study [J]. Stem Cell Res Ther. 2016; 7.

[17] Xiao Du, Xiaoping Chen, Kaiming Zhang, Haiyan Feng, Lei Wu. Experimental study on induced differentiation of human bone marrow mesenchymal stem cells into vascular endothelial cells in vitro. Chinese Journal of Integrative Medicine on Cardio-/Cerebrovascular Disease. 2009; 7 (8): 940-941.

[18] Jin LI. Effect of heat shock culture on FGFRs and apoptosis-related proteins of bone marrow mesenchymal stem cells transfected with bFGF [J]. Health standard management in China. 2018; 1: 134-137.

[19] Rong XU, Jinyong XU, Wei LIU. Experimental study on directional differentiation of Bone Marrow Mesenchymal Stem cells into Vascular Endothelial cells [J]. Journal of Biomedical Engineering. 2014; (2): 389-393.

[20] YANG Li-Xin, XU Zhi-Yun, Zhang Bao-Ren, Huang Sheng-Dong, LIU Yan-Ling. In vitro induction of bone marrow stromal stem cells to differentiate into endothelial cells [J]. Acad J Secil Med Univ, 2003; 24 (12): 1300-1304.

[21] Guan WANG, Jingya LI, Shengping YANG, Jianmin WANG. Induction of Astragalus Polysaccharide in differentiation of Bone Marrow Mesenchymal Stem cells into Neuron-like cells [J]. Research on Organization Engineering in China. 2015; (45) 7259-7262.

[22] $\mathrm{Wu} \mathrm{JX,} \mathrm{Xu} \mathrm{BL,} \mathrm{Huang} \mathrm{WL.} \mathrm{Advances} \mathrm{in} \mathrm{angiogenesis}$ inhibitors [J]. Cancer. 2005; 24 (3): 376-384.

[23] Bandara N, Gurusinghe S, Chen H, Chen S, Wang LX, Lim SY, Strappe P. Minicircle DNA-mediated endothelial nitric oxide synthase gene transfer enhances angiogenic responses of bone marrow-derived mesenchymal stemcells [J]. Stem Cell Res Ther. 2016; 7: 48doi: 10.1186/s13287-016-0307-2.

[24] Baoming Zhao, Fengliang Li, Ximing Song, et al. Effects of glutathione on the secretion of prostaglandin and endothelin-1 in human umbilical vein endothelial cells in the glycochemical end-product environment $[\mathrm{J}]$. Journal of Zhengzhou university (medical edition), 2011, 46 (4): 599-601.

[25] Groop PH, Forsblom C, Thomas MC. Mechanisms of disease: Pathway-selective insulin resistance and microvascular complications of diabetes [J]. Nat Clin Pract Endocrinol Metab. 2005; 1 (2): 100.

[26] Keliang Xie. Research progress of thrombosis and hemostasis test [J]. Medical information. 2014; 14: 655-656.

[27] Shen ling. Intervention of astragalusmembranaceus on differentiation and migration of human bone marrow mesenchymal stem cells induced by vascular endothelial growth factor/stromal cell-derived factor-1 in hypoxic environment [D]. Beijing University of Chinese Medicine. 2010 .

[28] Argyris T, Paschalis N, Andreas K, et al. Expression of Hypoxia-Inducible Factor (HIF)-1a-Vascular Endothelial Growth Factor (VEGF)-Inhibitory Growth Factor (ING)-4axis in sarcoidosis patients [J]. BMC Research Notes, 2012; 5 (1): $1-9$.

[29] Tao Y, Michael R, Soonpa M H, et al. Cardiac Engraftment of Genetically-Selected Parthenogenetic Stem Cell-Derived Cardiomyocytes [J]. PLOS ONE. 2015; 10 (6): e0131511.

[30] Ikhapoh Attairu Izuagie, Christopher J Pelham, Devendra K Agrawal. Synergistic effect of angiotensin II on vascular endothelial growth factor-A-mediated differentiation of bone marrow-derived mesenchymal stem cells into endothelial cells [J]. Stem Cell Res Ther. 2015; 6.

[31] Semenza GL. Hypoxia-inducible factors in physiology and medicine. Cell. 2012; 148 (3): 399-408.

[32] Jingwen Si, Ning Wang, Huan Wang, Juan Xie, Jian Yang, Hui $\mathrm{Yi}$, et al. HIF-1 $\alpha$ signaling activation by post-ischemia treatment with Astragaloside IV attenuates myocardial ischemia-reperfusion injury. PLOS ONE. 2014; 9 (9): e107832.

[33] Rissanen TT, Vajanto I, Hiltunen MO, et al. Expression of vascular endothelial growth factor and vascular endothelial growth factor receptor-2 (KDR/Flk-1) in ischemic skeletal muscle and its regeneration. Am J Pathol. 2002; 160: 1393-403.

[34] Tuomisto TT, Rissanen TT, Korkeela A, et al. HIF-VEGFVEGFR-2, TNF-a and IGF pathways are upregulated in critical human skeletal muscle ischemia as studied with DNA array. Atherosclerosis. 2004; 174: 111-20. 